\title{
Compound Option Pricing under Fuzzy Environment
}

\author{
Xiandong Wang, ${ }^{1,2}$ Jianmin $\mathrm{He}^{2}$ and Shouwei $\mathrm{Li}^{2}$ \\ ${ }^{1}$ School of Sciences, Changzhou Institute of Technology, Changzhou, Jiangsu 213002, China \\ ${ }^{2}$ School of Economics and Management, Southeast University, Nanjing, Jiangsu 211189, China \\ Correspondence should be addressed to Xiandong Wang; wxd.06@163.com
}

Received 5 October 2013; Revised 31 December 2013; Accepted 14 January 2014; Published 5 March 2014

Academic Editor: Francis T. K. Au

Copyright (C) 2014 Xiandong Wang et al. This is an open access article distributed under the Creative Commons Attribution License, which permits unrestricted use, distribution, and reproduction in any medium, provided the original work is properly cited.

\begin{abstract}
Considering the uncertainty of a financial market includes two aspects: risk and vagueness; in this paper, fuzzy sets theory is applied to model the imprecise input parameters (interest rate and volatility). We present the fuzzy price of compound option by fuzzing the interest and volatility in Geske's compound option pricing formula. For each $\alpha$, the $\alpha$-level set of fuzzy prices is obtained according to the fuzzy arithmetics and the definition of fuzzy-valued function. We apply a defuzzification method based on crisp possibilistic mean values of the fuzzy interest rate and fuzzy volatility to obtain the crisp possibilistic mean value of compound option price. Finally, we present a numerical analysis to illustrate the compound option pricing under fuzzy environment.
\end{abstract}

\section{Introduction}

Compound options are options with other options as underlying assets. Since Geske [1] derived the closed form pricing formula using the method of partial differential equations for the first time, some scholars have extended the pricing model and proposed some new pricing methods. For example, [2] used the martingale approach and the expectation of a truncated bivariate normal variables to prove the pricing formula for 2-fold compound options, respectively. The work of [3] extended the Geske model to a multivariate normal integral for the valuation of a compound real option. The work of [46 ] extended the Geske model to n-fold compound options. The work of $[7,8]$ introduced time dependent volatility and an interest rate to the pricing model of compound options. Fouque and Han [9] proposed perturbation approximation to compute the prices of compound options. There are few literature studied compound option pricing under jumpdiffusion model, stochastic volatility model, or stochastic interest rate model, such as [10-12]. Compound option is widely employed in the field of financial derivatives pricing, for instance, American put option [13], sequential exchange options [14], and sequential American exchange property options [15]. Compound option is also widely used in the real options; examples include project valuation of new drug application [16], valuation of multistage BOT projects [17], and decision-making in petroleum exploration [18].

The literature mentioned above studied compound option within stochastic framework. The uncertainty of the financial market includes two aspects: risk and vagueness, and the two parts could not substitute each other. In the real financial market, due to market fluctuations and human errors, some parameters such as the interest rate and volatility sometimes cannot be recorded or collected precisely. The risk uncertainty could be modeled by the probability theory; the vagueness could be modeled by a fuzzy methodology, the fuzzy sets theory provides an appropriate tool for tackling this kind of uncertainty. Therefore, the fuzzy sets theory proposed by Zadeh [19] has been widely used in the option pricing recently. The existing literature on option pricing under the fuzzy stochastic model mainly studied the European option, based on the Black-Scholes model. For example, Yoshida [20] introduced fuzzy logic to the stochastic financial model and discussed the valuation of the European options with the uncertainty of both randomness and fuzziness. Wu [21] considered the fuzzy pattern of the Black-Scholes formula by fuzzing the interest rate, volatility, and stock price in his paper when the arithmetics in the Black-Scholes formula are replaced by fuzzy arithmetic. The work of $[22,23]$ presented a sensitivity analysis based on the Black-Scholes formula. 
The work of [24] introduced a crisp weighted possibilistic mean value Black-Scholes option pricing formula. There are only few papers that studied American options or exotic options pricing within the Black-Scholes framework, such as [25-27], and few papers for alternative models with jumps [28-30]. As far as we know, there is no literature research on compound option pricing under fuzzy environment; this paper will consider both the risk and vagueness to study compound option pricing. The main contribution of this paper is that we present the $\alpha$-level set of fuzzy prices for each $\alpha$ and give a sensitivity analysis of the crisp possibilistic mean value of compound option price with respect to the core value of fuzzy interest rate and fuzzy volatility.

The remainder of the paper is organized as follows. In Section 2, the notions of fuzzy numbers and the arithmetics of fuzzy numbers are introduced. In Section 3, the pricing formula for compound option under stochastic model is introduced. Section 4 presents the fuzzy price, $\alpha$-level set of fuzzy prices, and the crisp possibilistic mean value of compound option price. In Section 5, a numerical analysis is performed. Finally, the conclusions are stated in Section 6.

\section{Fuzzy Numbers}

In this section we follow the notations and concepts introduced in $\mathrm{Wu}[21,31]$.

Let $\mathbb{R}$ be the set of all real numbers. Then a fuzzy subset $\widetilde{A}$ of $\mathbb{R}$ is defined by its membership function $\mu_{\widetilde{A}}: \mathbb{R} \rightarrow[0,1]$. We denote by $\widetilde{A}_{\alpha}=\left\{x: \mu_{\widetilde{A}}(x) \geq \alpha\right\}$ the $\alpha$-level set of $\widetilde{A}$ for all $\alpha \in(0,1]$. The 0 -level set $\widetilde{A}_{0}$ of $\widetilde{A}$ is defined by the closure of the set $\left\{x: \mu_{\widetilde{A}}(x)>0\right\} . \widetilde{A}$ is called a normal fuzzy set if there exists an $x$ such that $\mu_{\widetilde{A}}(x)=1$, and $\widetilde{A}$ is called a convex fuzzy set if $\mu_{\widetilde{A}}(\lambda x+(1-\lambda) y) \geq \min \left\{\mu_{\widetilde{A}}(x), \mu_{\widetilde{A}}(y)\right\}$ for all $\lambda \in[0,1]$.

Let $f$ be a real-valued function defined on $\mathbb{R}$. Then $f$ is said to be upper semicontinuous if $\{x: f(x) \geq \alpha\}$ is a closed set for each $\alpha$.

Let $\widetilde{a}$ be a fuzzy subset of $\mathbb{R}$. Then $\widetilde{a}$ is called a fuzzy number if the following conditions are satisfied:

(i) $\tilde{a}$ is a normal and convex fuzzy set;

(ii) its membership function $\mu_{\tilde{a}}$ is upper semicontinuous;

(iii) the 0-level set $\tilde{a}_{0}$ is bounded.

If $\widetilde{a}$ is a fuzzy number, then the $\alpha$-level set $\tilde{a}_{\alpha}$ is a closed interval and the $\alpha$-level set of $\widetilde{a}$ is denoted by $\tilde{a}_{\alpha}=\left[\tilde{a}_{\alpha}^{L}, \widetilde{a}_{\alpha}^{U}\right]$.

Lemma 1. Let $\tilde{a}$ and $\widetilde{b}$ be two fuzzy numbers. Then $\tilde{a} \oplus \widetilde{b}, \widetilde{a} \ominus \widetilde{b}$, and $\tilde{a} \otimes \widetilde{b}$ are also fuzzy numbers and their $\alpha$-level sets are

$$
\begin{gathered}
(\tilde{a} \oplus \widetilde{b})_{\alpha}=\left[\tilde{a}_{\alpha}^{L}+\widetilde{b}_{\alpha}^{L}, \widetilde{a}_{\alpha}^{U}+\widetilde{b}_{\alpha}^{U}\right], \\
(\tilde{a} \ominus \widetilde{b})_{\alpha}=\left[\widetilde{a}_{\alpha}^{L}-\widetilde{b}_{\alpha}^{U}, \widetilde{a}_{\alpha}^{U}-\widetilde{b}_{\alpha}^{L}\right], \\
(\tilde{a} \otimes \widetilde{b})_{\alpha}=\left[\min \left\{\tilde{a}_{\alpha}^{L} \widetilde{b}_{\alpha}^{L}, \widetilde{a}_{\alpha}^{L} \widetilde{b}_{\alpha}^{U}, \widetilde{a}_{\alpha}^{U} \widetilde{b}_{\alpha}^{L}, \widetilde{a}_{\alpha}^{U} \widetilde{b}_{\alpha}^{U}\right\},\right. \\
\left.\max \left\{\widetilde{a}_{\alpha}^{L} \widetilde{b}_{\alpha}^{L}, \tilde{a}_{\alpha}^{L} \widetilde{b}_{\alpha}^{U}, \widetilde{a}_{\alpha}^{U} \widetilde{b}_{\alpha}^{L}, \widetilde{a}_{\alpha}^{U} \widetilde{b}_{\alpha}^{U}\right\}\right],
\end{gathered}
$$

for all $\alpha \in[0,1]$. If the $\alpha$-level set $\widetilde{b}_{\alpha}$ of $\tilde{b}$ does not contain zero, then $\widetilde{a} \oslash \widetilde{b}$ is also a fuzzy number, and its $\alpha$-level set is

$$
\begin{array}{r}
(\tilde{a} \oslash \tilde{b})_{\alpha}=\left[\min \left\{\frac{\tilde{a}_{\alpha}^{L}}{\widetilde{b}_{\alpha}^{L}}, \frac{\tilde{a}_{\alpha}^{L}}{\widetilde{b}_{\alpha}^{U}}, \frac{\tilde{a}_{\alpha}^{U}}{\widetilde{b}_{\alpha}^{L}}, \frac{\widetilde{a}_{\alpha}^{U}}{\widetilde{b}_{\alpha}^{U}}\right\},\right. \\
\left.\max \left\{\frac{\tilde{a}_{\alpha}^{L}}{\widetilde{b}_{\alpha}^{L}}, \frac{\tilde{a}_{\alpha}^{L}}{\widetilde{b}_{\alpha}^{U}}, \frac{\widetilde{a}_{\alpha}^{U}}{\widetilde{b}_{\alpha}^{L}}, \frac{\widetilde{a}_{\alpha}^{U}}{\widetilde{b}_{\alpha}^{U}}\right\}\right],
\end{array}
$$

for all $\alpha \in[0,1]$.

$\tilde{a}$ is called a crisp number with value $m$ if its membership function is

$$
\mu_{\tilde{a}}(x)= \begin{cases}1 & \text { if } x=m, \\ 0 & \text { otherwise }\end{cases}
$$

It is denoted by $\tilde{a}=\tilde{1}_{\{m\}}$. We see that the real numbers are the special case of the fuzzy numbers when the real numbers are regarded as the crisp numbers.

Lemma 2. Let $f\left(x_{1}, x_{2}, \ldots, x_{n}\right)$ be a continuous real-valued function defined on $\mathbb{R}^{n}$ and let $\tilde{a}_{1}, \tilde{a}_{2}, \ldots, \tilde{a}_{n}$ be $n$ fuzzy numbers. Let $\tilde{f}: \mathscr{F}^{n} \rightarrow \mathscr{F}$ be a fuzzy-valued function induced by $f\left(x_{1}, x_{2}, \ldots, x_{n}\right)$ via the extension principle. Suppose that each $\left\{\left(x_{1}, x_{2}, \ldots, x_{n}\right): y=f\left(x_{1}, x_{2}, \ldots, x_{n}\right)\right\}$ is a compact subset of $\mathbb{R}^{n}$ for $y$ in the range of $f$. Then $\tilde{f}\left(\widetilde{a}_{1}, \tilde{a}_{2}, \ldots, \widetilde{a}_{n}\right)$ is a fuzzy number and its $\alpha$-level set is

$$
\begin{aligned}
(\tilde{f} & \left.\left(\widetilde{a}_{1}, \tilde{a}_{2}, \ldots, \tilde{a}_{n}\right)\right)_{\alpha} \\
= & \left\{f\left(x_{1}, x_{2}, \ldots, x_{n}\right): x_{1} \in\left(\widetilde{a}_{1}\right)_{\alpha}, \ldots, x_{n} \in\left(\widetilde{a}_{n}\right)_{\alpha}\right\} \\
= & \left\{f\left(x_{1}, x_{2}, \ldots, x_{n}\right):\left(\widetilde{a}_{1}\right)_{\alpha}^{L} \leq x_{1} \leq\left(\widetilde{a}_{1}\right)_{\alpha}^{U}, \ldots,\right. \\
& \left.\left(\tilde{a}_{n}\right)_{\alpha}^{L} \leq x_{n} \leq\left(\tilde{a}_{n}\right)_{\alpha}^{U}\right\} .
\end{aligned}
$$

The membership function of a triangular fuzzy number $\tilde{a}$ is defined by

$$
\mu_{\tilde{a}}(x)= \begin{cases}1-\frac{a_{c}-x}{\gamma} & \text { if } a_{c}-\gamma \leq x \leq a_{c}, \\ 1-\frac{x-a_{c}}{\beta} & \text { if } a_{c} \leq x \leq a_{c}+\beta, \\ 0 & \text { otherwise }\end{cases}
$$

which is denoted by $\tilde{a}=\left(a_{c}-\gamma, a_{c}, a_{c}+\beta\right), a_{c}$ is called the core value of $\tilde{a}$, and $\gamma>0$ and $\beta>0$ are called the left and right spreads of $\tilde{a}$, respectively. The triangular fuzzy number $\tilde{a}$ can be expressed as "around $a_{c}$ " or "being approximately equal to $a_{c}$ " "The $\alpha$-level set of $\widetilde{a}$ is $\widetilde{a}_{\alpha}=\left[a_{c}-(1-\alpha) \gamma, a_{c}+(1-\alpha) \beta\right]$.

Fullér and Majlender [32] defined the crisp possibilistic mean value of a fuzzy number $\tilde{a}$ with $\alpha$-level set $\tilde{a}_{\alpha}=\left[\widetilde{a}_{\alpha}^{L}, \widetilde{a}_{\alpha}^{U}\right]$ as

$$
M(\widetilde{a})=\int_{0}^{1} \alpha\left(\widetilde{a}_{\alpha}^{L}+\widetilde{a}_{\alpha}^{U}\right) d \alpha .
$$

The crisp possibilistic mean value of a triangular fuzzy number $\widetilde{a}=\left(a_{c}-\gamma, a_{c}, a_{c}+\beta\right)$ is $M(\widetilde{a})=a_{c}+(\beta-\gamma) / 6$. 


\section{Compound Option Pricing under Stochastic Model}

A compound option is an option on an option. Therefore, a compound option has two expiration dates and two strike prices. There are four types of European compound options; in this paper we take a call on a call as an example. If an investor buys a compound option at time 0 , then, on the first expiration date $T_{1}$, the option holder has the right to buy a new call option with the strike price $K_{1}$. The new option gives the holder the right to buy the underlying asset with the strike price $K_{2}$ at time $T_{2}$.

We suppose the underlying asset price $S(t)$ satisfies the following stochastic differential equation:

$$
d S(t)=S(t)[\mu d t+\sigma d W(t)]
$$

where $\mu$ is the expected rate of return, $\sigma$ is the volatility, and $W(t)$ is a standard Brownian motion. The well-known closed form compound option pricing formula is presented in Geske [1] as the following lemma.

Lemma 3. The closed form pricing formula for compound option at time 0 is

$$
C=S N_{2}\left(d_{1}, d_{2}, \rho\right)-K_{2} \mathrm{e}^{-r T_{2}} N_{2}\left(d_{3}, d_{4}, \rho\right)-K_{1} \mathrm{e}^{-r T_{1}} N\left(d_{3}\right),
$$

where

$$
\begin{gathered}
d_{1}=\frac{\ln \left(S / S_{*}\right)+\left(r+(1 / 2) \sigma^{2}\right) T_{1}}{\sigma \sqrt{T_{1}}}, \\
d_{2}=\frac{\ln \left(S / K_{2}\right)+\left(r+(1 / 2) \sigma^{2}\right) T_{2}}{\sigma \sqrt{T_{2}}}, \\
d_{3}=d_{1}-\sigma \sqrt{T_{1}}, \\
d_{4}=d_{2}-\sigma \sqrt{T_{2}}, \\
\rho=\sqrt{\frac{T_{1}}{T_{2}}} .
\end{gathered}
$$

$r$ is the risk-free interest rate, $S$ is the underlying asset price at time $0, N(x)$ is the standard normal distribution function, $N_{2}(x, y, \rho)$ is the bivariate standard normal distribution function with $x$ and $y$ as upper limits and $\rho$ as the correlation coefficient between the two variables, and $S_{*}$ is the unique solution of the equation

$$
x N\left(d+\sigma \sqrt{T_{2}-T_{1}}\right)-K_{2} \mathrm{e}^{-r\left(T_{2}-T_{1}\right)} N(d)=K_{1},
$$

where

$$
d=\frac{\ln \left(x / K_{2}\right)+\left(r-(1 / 2) \sigma^{2}\right)\left(T_{2}-T_{1}\right)}{\sigma \sqrt{T_{2}-T_{1}}} .
$$

\section{Compound Option Pricing under Fuzzy Environment}

As discussed before, owing to the imprecise information and the fluctuations of the financial market from time to time, it is unreasonable to assume that the interest rate $r$ and the volatility $\sigma$ are constants. According to $\mathrm{Wu}$ [21] and Nowak and Romaniuk [29], we replace $r$ and $\sigma$ in (8) by fuzzy numbers $\widetilde{r}$ and $\widetilde{\sigma}$, respectively, and replace the arithmetics by fuzzy arithmetics to get the analytical formula for the compound option under fuzzy environment, which is given in Theorem 4.

Theorem 4. Let the interest rate and the volatility be fuzzy numbers. Then the fuzzy price of compound option is

$$
\begin{aligned}
\widetilde{C}= & \widetilde{1}_{\{S\}} \otimes \widetilde{N}_{2}\left(\widetilde{d}_{1}, \widetilde{d}_{2}, \widetilde{1}_{\{\rho\}}\right) \odot \widetilde{1}_{\left\{K_{2}\right\}} \otimes \mathrm{e}^{-\tilde{r} \otimes \tilde{1}_{\left\{T_{2}\right\}}} \\
& \otimes \widetilde{N}_{2}\left(\widetilde{d}_{3}, \widetilde{d}_{4}, \widetilde{1}_{\{\rho\}}\right) \odot \widetilde{1}_{\left\{K_{1}\right\}} \otimes \mathrm{e}^{-\widetilde{r} \otimes \tilde{1}_{\left\{T_{1}\right\}}} \otimes \widetilde{N}\left(\widetilde{d}_{3}\right),
\end{aligned}
$$

where

$$
\begin{aligned}
& \tilde{d}_{1}=\left[\tilde{1}_{\left\{\ln \left(S / S^{*}\right)\right\}} \oplus\left(\tilde{r} \oplus \tilde{1}_{\{1 / 2\}} \otimes \widetilde{\sigma} \otimes \widetilde{\sigma}\right) \otimes \tilde{1}_{\left\{T_{1}\right\}}\right] \\
& \oslash\left(\widetilde{\sigma} \otimes \widetilde{1}_{\left\{\sqrt{T_{1}}\right\}}\right), \\
& \widetilde{d}_{2}=\left[\tilde{1}_{\left\{\ln \left(S / K_{2}\right)\right\}} \oplus\left(\tilde{r} \oplus \tilde{1}_{\{1 / 2\}} \otimes \widetilde{\sigma} \otimes \widetilde{\sigma}\right) \otimes \tilde{1}_{\left\{T_{2}\right\}}\right] \\
& \oslash\left(\tilde{\sigma} \otimes \tilde{1}_{\left\{\sqrt{T_{2}}\right\}}\right), \\
& \widetilde{d}_{3}=\widetilde{d}_{1} \ominus\left(\widetilde{\sigma} \otimes \widetilde{1}_{\left\{\sqrt{T_{1}}\right\}}\right), \\
& \widetilde{d}_{4}=\widetilde{d}_{2} \ominus\left(\widetilde{\sigma} \otimes \widetilde{1}_{\left\{\sqrt{T_{2}}\right\}}\right) \text {. }
\end{aligned}
$$

$S^{*}$ is the unique solution of the equation

$$
x N\left(d^{*}+M(\widetilde{\sigma}) \sqrt{T_{2}-T_{1}}\right)-K_{2} \mathrm{e}^{-M(\widetilde{r})\left(T_{2}-T_{1}\right)} N\left(d^{*}\right)=K_{1},
$$

where

$$
\begin{gathered}
d^{*}=\frac{\ln \left(x / K_{2}\right)+\left(M(\widetilde{r})-(1 / 2)[M(\widetilde{\sigma})]^{2}\right)\left(T_{2}-T_{1}\right)}{M(\widetilde{\sigma}) \sqrt{T_{2}-T_{1}}}, \\
M(\widetilde{r})=\int_{0}^{1} \alpha\left(\widetilde{r}_{\alpha}^{L}+\widetilde{r}_{\alpha}^{U}\right) d \alpha \\
M(\widetilde{\sigma})=\int_{0}^{1} \alpha\left(\widetilde{\sigma}_{\alpha}^{L}+\widetilde{\sigma}_{\alpha}^{U}\right) d \alpha .
\end{gathered}
$$

$\widetilde{r}_{\alpha}^{L}$ and $\widetilde{r}_{\alpha}^{U}$ are the left-end point and right-end point of the $\alpha$ level set of $\widetilde{r}$, respectively, and $\widetilde{\sigma}_{\alpha}^{L}$ and $\widetilde{\sigma}_{\alpha}^{U}$ are the left-end point and right-end point of the $\alpha$-level set of $\widetilde{\sigma}$, respectively.

Proof. It is easily obtained from Lemma 3 by replacing $r$ and $\sigma$ by the fuzzy numbers $\widetilde{r}$ and $\widetilde{\sigma}$, respectively, and replacing the arithmetics by fuzzy arithmetics.

Under fuzzy environment, the option price $\widetilde{C}$ is a fuzzy number, the $\alpha$-level set of $\widetilde{C}$ may be denoted as $\widetilde{C}_{\alpha}=\left[\widetilde{C}_{\alpha}^{L}, \widetilde{C}_{\alpha}^{U}\right]$, and $\widetilde{C}_{\alpha}^{L}$ and $\widetilde{C}_{\alpha}^{U}$ can be calculated as the following theorem. 
Theorem 5. The left-end point and right-end point of the $\alpha$ level set $\widetilde{C}_{\alpha}=\left[\widetilde{C}_{\alpha}^{L}, \widetilde{C}_{\alpha}^{U}\right]$ can be calculated as follows:

$$
\begin{aligned}
\widetilde{C}_{\alpha}^{L}= & S N_{2}\left(\left(\tilde{d}_{1}\right)_{\alpha}^{L},\left(\tilde{d}_{2}\right)_{\alpha}^{L}, \rho\right)-K_{2} \mathrm{e}^{-\widetilde{r}_{\alpha}^{L} T_{2}} N_{2}\left(\left(\tilde{d}_{3}\right)_{\alpha}^{U},\left(\tilde{d}_{4}\right)_{\alpha}^{U}, \rho\right) \\
& -K_{1} \mathrm{e}^{-\tilde{r}_{\alpha}^{L} T_{1}} N\left(\left(\widetilde{d}_{3}\right)_{\alpha}^{U}\right), \\
\widetilde{C}_{\alpha}^{U}= & S N_{2}\left(\left(\tilde{d}_{1}\right)_{\alpha}^{U},\left(\tilde{d}_{2}\right)_{\alpha}^{U}, \rho\right)-K_{2} \mathrm{e}^{-\widetilde{r}_{\alpha}^{U} T_{2}} N_{2}\left(\left(\tilde{d}_{3}\right)_{\alpha}^{L},\left(\tilde{d}_{4}\right)_{\alpha}^{L}, \rho\right) \\
& -K_{1} \mathrm{e}^{-\widetilde{r}_{\alpha}^{U} T_{1}} N\left(\left(\widetilde{d}_{3}\right)_{\alpha}^{L}\right),
\end{aligned}
$$

where

$$
\begin{aligned}
& \left(\widetilde{d}_{1}\right)_{\alpha}^{L}=\min \left\{\frac{\widetilde{a}_{\alpha}^{L}}{\widetilde{b}_{\alpha}^{L}}, \frac{\widetilde{a}_{\alpha}^{L}}{\widetilde{b}_{\alpha}^{U}}, \frac{\widetilde{a}_{\alpha}^{U}}{\widetilde{b}_{\alpha}^{L}}, \frac{\widetilde{a}_{\alpha}^{U}}{\widetilde{b}_{\alpha}^{U}}\right\}, \\
& \left(\widetilde{d}_{1}\right)_{\alpha}^{U}=\max \left\{\frac{\widetilde{a}_{\alpha}^{L}}{\widetilde{b}_{\alpha}^{L}}, \frac{\widetilde{a}_{\alpha}^{L}}{\widetilde{b}_{\alpha}^{U}}, \frac{\widetilde{a}_{\alpha}^{U}}{\widetilde{b}_{\alpha}^{L}}, \frac{\widetilde{a}_{\alpha}^{U}}{\widetilde{b}_{\alpha}^{U}}\right\}, \\
& \tilde{a}_{\alpha}^{L}=\ln \frac{S}{S^{*}}+\left[\widetilde{r}_{\alpha}^{L}+\frac{1}{2}\left(\widetilde{\sigma}_{\alpha}^{L}\right)^{2}\right] T_{1} \text {, } \\
& \widetilde{a}_{\alpha}^{U}=\ln \frac{S}{S^{*}}+\left[\widetilde{r}_{\alpha}^{U}+\frac{1}{2}\left(\widetilde{\sigma}_{\alpha}^{U}\right)^{2}\right] T_{1} \text {, } \\
& \tilde{b}_{\alpha}^{L}=\widetilde{\sigma}_{\alpha}^{L} \sqrt{T_{1}}, \quad \tilde{b}_{\alpha}^{U}=\widetilde{\sigma}_{\alpha}^{U} \sqrt{T_{1}} \text {, } \\
& \left(\widetilde{d}_{2}\right)_{\alpha}^{L}=\min \left\{\frac{\widetilde{m}_{\alpha}^{L}}{\widetilde{n}_{\alpha}^{L}}, \frac{\widetilde{m}_{\alpha}^{L}}{\tilde{n}_{\alpha}^{U}}, \frac{\widetilde{m}_{\alpha}^{U}}{\widetilde{n}_{\alpha}^{L}}, \frac{\widetilde{m}_{\alpha}^{U}}{\widetilde{n}_{\alpha}^{U}}\right\}, \\
& \left(\widetilde{d}_{2}\right)_{\alpha}^{U}=\max \left\{\frac{\widetilde{m}_{\alpha}^{L}}{\widetilde{n}_{\alpha}^{L}}, \frac{\widetilde{m}_{\alpha}^{L}}{\widetilde{n}_{\alpha}^{U}}, \frac{\widetilde{m}_{\alpha}^{U}}{\widetilde{n}_{\alpha}^{L}}, \frac{\widetilde{m}_{\alpha}^{U}}{\widetilde{n}_{\alpha}^{U}}\right\}, \\
& \widetilde{m}_{\alpha}^{L}=\ln \frac{S}{K_{2}}+\left[\widetilde{r}_{\alpha}^{L}+\frac{1}{2}\left(\widetilde{\sigma}_{\alpha}^{L}\right)^{2}\right] T_{2} \text {, } \\
& \widetilde{m}_{\alpha}^{U}=\ln \frac{S}{K_{2}}+\left[\widetilde{r}_{\alpha}^{U}+\frac{1}{2}\left(\tilde{\sigma}_{\alpha}^{U}\right)^{2}\right] T_{2}, \\
& \tilde{n}_{\alpha}^{L}=\tilde{\sigma}_{\alpha}^{L} \sqrt{T_{2}}, \quad \tilde{n}_{\alpha}^{U}=\tilde{\sigma}_{\alpha}^{U} \sqrt{T_{2}}, \\
& \left(\tilde{d}_{3}\right)_{\alpha}^{L}=\left(\tilde{d}_{1}\right)_{\alpha}^{L}-\widetilde{\sigma}_{\alpha}^{U} \sqrt{T_{1}}, \\
& \left(\tilde{d}_{3}\right)_{\alpha}^{U}=\left(\tilde{d}_{1}\right)_{\alpha}^{U}-\tilde{\sigma}_{\alpha}^{L} \sqrt{T_{1}}, \\
& \left(\tilde{d}_{4}\right)_{\alpha}^{L}=\left(\widetilde{d}_{2}\right)_{\alpha}^{L}-\widetilde{\sigma}_{\alpha}^{U} \sqrt{T_{2}}, \\
& \left(\tilde{d}_{4}\right)_{\alpha}^{U}=\left(\tilde{d}_{2}\right)_{\alpha}^{U}-\widetilde{\sigma}_{\alpha}^{L} \sqrt{T_{2}} .
\end{aligned}
$$

Proof. Because the standard normal distribution function $N(x)$ and the bivariate standard normal distribution function $N(x, y, \rho)$ are increasing functions, $\mathrm{e}^{-x}$ is a decreasing function; according to Lemmas 1 and 2, from (12) the left-end point of the $\alpha$-level set $\widetilde{C}_{\alpha}=\left[\widetilde{C}_{\alpha}^{L}, \widetilde{C}_{\alpha}^{U}\right]$ is

$$
\begin{aligned}
& \widetilde{C}_{\alpha}^{L}=\left(\widetilde{1}_{\{S\}} \otimes \widetilde{N}_{2}\left(\widetilde{d}_{1}, \widetilde{d}_{2}, \widetilde{1}_{\{\rho\}}\right)\right)_{\alpha}^{L} \\
& -\left(\tilde{1}_{\left\{K_{2}\right\}} \otimes \mathrm{e}^{-\tilde{r} \otimes \tilde{1}_{\left\{T_{2}\right\}}} \otimes \widetilde{N}_{2}\left(\tilde{d}_{3}, \tilde{d}_{4}, \tilde{1}_{\{\rho\}}\right)\right)_{\alpha}^{U} \\
& -\left(\widetilde{1}_{\left\{K_{1}\right\}} \otimes \mathrm{e}^{-\tilde{r} \otimes \tilde{1}_{\left\{T_{1}\right\}}} \otimes \widetilde{N}\left(\widetilde{d}_{3}\right)\right)_{\alpha}^{U} \\
& =S N_{2}\left(\left(\widetilde{d}_{1}\right)_{\alpha}^{L},\left(\widetilde{d}_{2}\right)_{\alpha}^{L}, \rho\right)-K_{2} \mathrm{e}^{-\widetilde{r}_{\alpha}^{L} T_{2}} N_{2}\left(\left(\widetilde{d}_{3}\right)_{\alpha}^{U},\left(\widetilde{d}_{4}\right)_{\alpha}^{U}, \rho\right) \\
& -K_{1} \mathrm{e}^{-\widetilde{r}_{\alpha}^{L} T_{1}} N\left(\left(\tilde{d}_{3}\right)_{\alpha}^{U}\right)
\end{aligned}
$$

where $\left(\tilde{d}_{1}\right)_{\alpha}^{L},\left(\tilde{d}_{2}\right)_{\alpha}^{L},\left(\tilde{d}_{3}\right)_{\alpha}^{U}$, and $\left(\tilde{d}_{4}\right)_{\alpha}^{U}$ are given as Theorem 5 . In the same way, $\widetilde{C}_{\alpha}^{U}$ can be proved.

We provide a method to find a crisp number that synthesizes the fuzzy number by replacing $\widetilde{r}$ and $\widetilde{\sigma}$ in Theorem 4 by their crisp possibilistic mean values $M(\widetilde{r})$ and $M(\widetilde{\sigma})$, respectively, to get the crisp possibilistic mean value of compound option price.

Theorem 6. The crisp possibilistic mean value of the compound option price is

$$
\begin{aligned}
M(\widetilde{C})= & S N_{2}\left(M\left(\tilde{d}_{1}\right), M\left(\widetilde{d}_{2}\right), \rho\right) \\
& -K_{2} \mathrm{e}^{-M(\widetilde{r}) T_{2}} N_{2}\left(M\left(\widetilde{d}_{3}\right), M\left(\widetilde{d}_{4}\right), \rho\right) \\
& -K_{1} \mathrm{e}^{-M(\widetilde{r}) T_{1}} N\left(M\left(\tilde{d}_{3}\right)\right),
\end{aligned}
$$

where

$$
\begin{gathered}
M\left(\tilde{d}_{1}\right)=\frac{\ln \left(S / S^{*}\right)+\left(M(\widetilde{r})+(1 / 2)[M(\widetilde{\sigma})]^{2}\right) T_{1}}{M(\widetilde{\sigma}) \sqrt{T_{1}}} \\
M\left(\widetilde{d}_{2}\right)=\frac{\ln \left(S / K_{2}\right)+\left(M(\widetilde{r})+(1 / 2)[M(\widetilde{\sigma})]^{2}\right) T_{2}}{M(\widetilde{\sigma}) \sqrt{T_{2}}} \\
M\left(\widetilde{d}_{3}\right)=M\left(\widetilde{d}_{1}\right)-M(\widetilde{\sigma}) \sqrt{T_{1}} \\
M\left(\tilde{d}_{4}\right)=M\left(\tilde{d}_{2}\right)-M(\widetilde{\sigma}) \sqrt{T_{2}} .
\end{gathered}
$$

Proof. From Theorem 4 we have

$$
\begin{aligned}
M(\widetilde{C})= & S M\left(\widetilde{N}_{2}\left(\widetilde{d}_{1}, \widetilde{d}_{2}, \widetilde{1}_{\{\rho\}}\right)\right) \\
& -K_{2} M\left(\mathrm{e}^{-\widetilde{r} \otimes \widetilde{1}_{\left\{T_{2}\right\}}}\right) M\left(\widetilde{N}_{2}\left(\widetilde{d}_{3}, \widetilde{d}_{4}, \widetilde{1}_{\{\rho\}}\right)\right) \\
& -K_{1} M\left(\mathrm{e}^{-\tilde{r} \otimes \tilde{1}_{\left\{T_{1}\right\}}}\right) M\left(\widetilde{N}\left(\widetilde{d}_{3}\right)\right)
\end{aligned}
$$


TABLE 1: The numerical results of $S_{*}$ and $C$ under Black-Scholes model.

\begin{tabular}{lcccccccc}
\hline$S$ & $K_{1}$ & $K_{2}$ & $T_{1}$ & $T_{2}$ & $r$ & $\sigma$ & $S_{*}$ & $C$ \\
\hline 90 & 5 & 90 & 0.5 & 1 & 0.05 & 0.3 & 82.8336 & 8.8868 \\
95 & 5 & 90 & 0.5 & 1 & 0.05 & 0.3 & 82.8336 & 11.8885 \\
105 & 5 & 90 & 0.5 & 1 & 0.05 & 0.3 & 82.8336 & 18.9879 \\
100 & 4 & 90 & 0.5 & 1 & 0.05 & 0.3 & 80.3707 & 16.0888 \\
100 & 4.5 & 90 & 0.5 & 1 & 0.05 & 0.3 & 81.6430 & 15.6766 \\
100 & 6 & 90 & 0.5 & 1 & 0.05 & 0.3 & 85.0172 & 14.4955 \\
100 & 5 & 80 & 0.5 & 1 & 0.05 & 0.3 & 74.8669 & 21.7684 \\
100 & 5 & 85 & 0.5 & 1 & 0.05 & 0.3 & 78.8616 & 18.3432 \\
100 & 5 & 95 & 0.5 & 1 & 0.05 & 0.3 & 86.7835 & 12.5739 \\
100 & 5 & 90 & 0.25 & 1 & 0.05 & 0.3 & 78.3652 & 14.8361 \\
100 & 5 & 90 & 0.55 & 1 & 0.05 & 0.3 & 83.8285 & 15.3843 \\
100 & 5 & 90 & 0.75 & 1 & 0.05 & 0.3 & 88.2795 & 15.8987 \\
100 & 5 & 90 & 0.5 & 0.75 & 0.05 & 0.3 & 88.2795 & 13.6596 \\
100 & 5 & 90 & 0.5 & 1 & 0.05 & 0.3 & 82.8336 & 15.2744 \\
100 & 5 & 90 & 0.5 & 1.25 & 0.05 & 0.3 & 78.3652 & 16.8882 \\
100 & 5 & 90 & 0.5 & 1 & 0.04 & 0.3 & 83.1909 & 14.7403 \\
100 & 5 & 90 & 0.5 & 1 & 0.055 & 0.3 & 82.6556 & 15.5449 \\
100 & 5 & 90 & 0.5 & 1 & 0.06 & 0.3 & 82.4780 & 15.8173 \\
100 & 5 & 90 & 0.5 & 1 & 0.05 & 0.25 & 85.4360 & 13.6727 \\
100 & 5 & 90 & 0.5 & 1 & 0.05 & 0.32 & 81.7635 & 15.9318 \\
100 & 5 & 90 & 0.5 & 1 & 0.05 & 0.35 & 80.1417 & 16.9306 \\
\hline & & & & & & & &
\end{tabular}

$$
\begin{aligned}
= & S N_{2}\left(M\left(\tilde{d}_{1}\right), M\left(\tilde{d}_{2}\right), \rho\right) \\
& -K_{2} \mathrm{e}^{-M(\tilde{r}) T_{2}} N_{2}\left(M\left(\widetilde{d}_{3}\right), M\left(\widetilde{d}_{4}\right), \rho\right) \\
& -K_{1} \mathrm{e}^{-M(\tilde{r}) T_{1}} N\left(M\left(\widetilde{d}_{3}\right)\right),
\end{aligned}
$$

where $M\left(\widetilde{d}_{i}\right), i=1,2,3,4$, are given as Theorem 6 .

\section{Numerical Analysis}

In this section, we provide some numerical results of compound option pricing under fuzzy environment. For comparison, Table 1 presents the numerical results of the critical value $S_{*}$ and compound option price $C$ under the BlackScholes model, where the critical value $S_{*}$ can be obtained by solving (10) using the bisection method, and the precision is $10^{-5}$. All the computations are performed using Matlab software.

Triangular fuzzy numbers are applied to denote the fuzzy parameters $\widetilde{r}$ and $\widetilde{\sigma}$ because of their simple properties. We take $\tilde{r}=\left(r_{c}-0.002, r_{c}, r_{c}+0.001\right)$, where $r_{c}$ is the core value of $\tilde{r}$ and the left and right spreads of $\tilde{r}$ are 0.002 and 0.001 , respectively, and $\widetilde{\sigma}=\left(\sigma_{c}-0.01, \sigma_{c}, \sigma_{c}+0.02\right)$, where $\sigma_{c}$ is the core value of $\widetilde{\sigma}$ and the left and right spreads of $\widetilde{\sigma}$ are 0.01 and 0.02 , respectively. Table 2 presents the critical value $S^{*}$ and the crisp possibilistic mean value of compound option price $M(\widetilde{C})$ under fuzzy environment, where the critical value $S^{*}$ can be obtained by solving (14) using the bisection method, and the precision is $10^{-5}$. For each match group the values of
TABLe 2: The numerical result of $S^{*}$ and $M(\widetilde{C})$ under the fuzzy environment.

\begin{tabular}{lcccccccc}
\hline$S$ & $K_{1}$ & $K_{2}$ & $T_{1}$ & $T_{2}$ & $r_{c}$ & $\sigma_{c}$ & $S^{*}$ & $M(\widetilde{C})$ \\
\hline 90 & 5 & 90 & 0.5 & 1 & 0.05 & 0.3 & 82.7509 & 8.9357 \\
95 & 5 & 90 & 0.5 & 1 & 0.05 & 0.3 & 82.7509 & 11.9369 \\
105 & 5 & 90 & 0.5 & 1 & 0.05 & 0.3 & 82.7509 & 19.0290 \\
100 & 4 & 90 & 0.5 & 1 & 0.05 & 0.3 & 80.2813 & 16.1338 \\
100 & 4.5 & 90 & 0.5 & 1 & 0.05 & 0.3 & 81.5571 & 15.7219 \\
100 & 6 & 90 & 0.5 & 1 & 0.05 & 0.3 & 84.9404 & 14.5412 \\
100 & 5 & 80 & 0.5 & 1 & 0.05 & 0.3 & 74.7967 & 21.7981 \\
100 & 5 & 85 & 0.5 & 1 & 0.05 & 0.3 & 78.7852 & 18.3814 \\
100 & 5 & 95 & 0.5 & 1 & 0.05 & 0.3 & 86.6946 & 12.6250 \\
100 & 5 & 90 & 0.25 & 1 & 0.05 & 0.3 & 78.2638 & 14.8803 \\
100 & 5 & 90 & 0.55 & 1 & 0.05 & 0.3 & 83.7505 & 15.4312 \\
100 & 5 & 90 & 0.75 & 1 & 0.05 & 0.3 & 88.2253 & 15.9460 \\
100 & 5 & 90 & 0.5 & 0.75 & 0.05 & 0.3 & 88.2253 & 13.7005 \\
100 & 5 & 90 & 0.5 & 1 & 0.05 & 0.3 & 82.7509 & 15.3199 \\
100 & 5 & 90 & 0.5 & 1.25 & 0.05 & 0.3 & 78.2638 & 16.9375 \\
100 & 5 & 90 & 0.5 & 1 & 0.04 & 0.3 & 83.1077 & 14.7869 \\
100 & 5 & 90 & 0.5 & 1 & 0.055 & 0.3 & 82.5732 & 15.5898 \\
100 & 5 & 90 & 0.5 & 1 & 0.06 & 0.3 & 82.3959 & 15.8616 \\
100 & 5 & 90 & 0.5 & 1 & 0.05 & 0.25 & 85.3576 & 13.7159 \\
100 & 5 & 90 & 0.5 & 1 & 0.05 & 0.32 & 81.6798 & 15.9782 \\
100 & 5 & 90 & 0.5 & 1 & 0.05 & 0.35 & 80.0567 & 16.9781 \\
\hline & & & & & & & &
\end{tabular}

parameters $S, K_{1}, K_{2}, T_{1}$, and $T_{2}$ in Table 2 are the same as those in Table 1 , and the cores $r_{c}$ and $\sigma_{c}$ are equal to $r$ and $\sigma$ in Table 1, respectively.

From Tables 1 and 2, the compound option prices derived from the Black-Scholes model are slightly lower than the prices derived from the crisp possibilistic mean value with the same parameters. This seems to be consistent with our intuition that the crisp possibilistic mean value model contains more uncertainty than the Black-Scholes model (see $[28,30])$. But this intuition is not necessarily true, which one is bigger between $C$ and $M(\widetilde{C})$ is related to the selected parameters. Similarly, from Tables 1 and 2, we notice that $S_{*}$ is slightly higher than $S^{*}$; this conclusion is not surely true. For example, when $S=100, K_{1}=5, K_{2}=90, T_{1}=0.5, T_{2}=1$, $\widetilde{r}=(0.049,0.05,0.052)$, and $\widetilde{\sigma}=(0.28,0.3,0.31)$, then the computing result is $S_{*}=82.8336, C=15.2744, S^{*}=82.9162$, and $M(\widetilde{C})=15.2290$; obviously, $S_{*}<S^{*}$ and $C>M(\widetilde{C})$.

In order to perform the sensitivity analysis we set our base case with $S=100, K_{1}=5, K_{2}=90, T_{1}=0.5, T_{2}=1$, $r_{c}=0.05$, and $\sigma_{c}=0.3$. Figures 1 to 7 give the sensitivity analysis of $S^{*}$ and $M(\widetilde{C})$ with respect to $S, K_{1}, K_{2}, T_{1}, T_{2}, r_{c}$, and $\sigma_{c}$, respectively. We can see that $S^{*}$ does not vary with the change of $S$, because (14) does not contain $S$. $S^{*}$ is increasing with respect to $K_{1}, K_{2}$, and $T_{1}$ and is decreasing with respect to $T_{2}, r_{c}$, and $\sigma_{c}$, because the value of call option increases with the increase of the stock price, interest rate, volatility and maturity time, and the value of call option falls with the increase of strike price; when the left and right spreads of $\widetilde{r}$ and $\widetilde{\sigma}$ are fixed (therefore, $M(\widetilde{r})$ and $M(\widetilde{\sigma})$ are increasing with respect to $r_{c}$ and $\sigma_{c}$, resp.) (14) means that $S^{*}$ is the underlying 


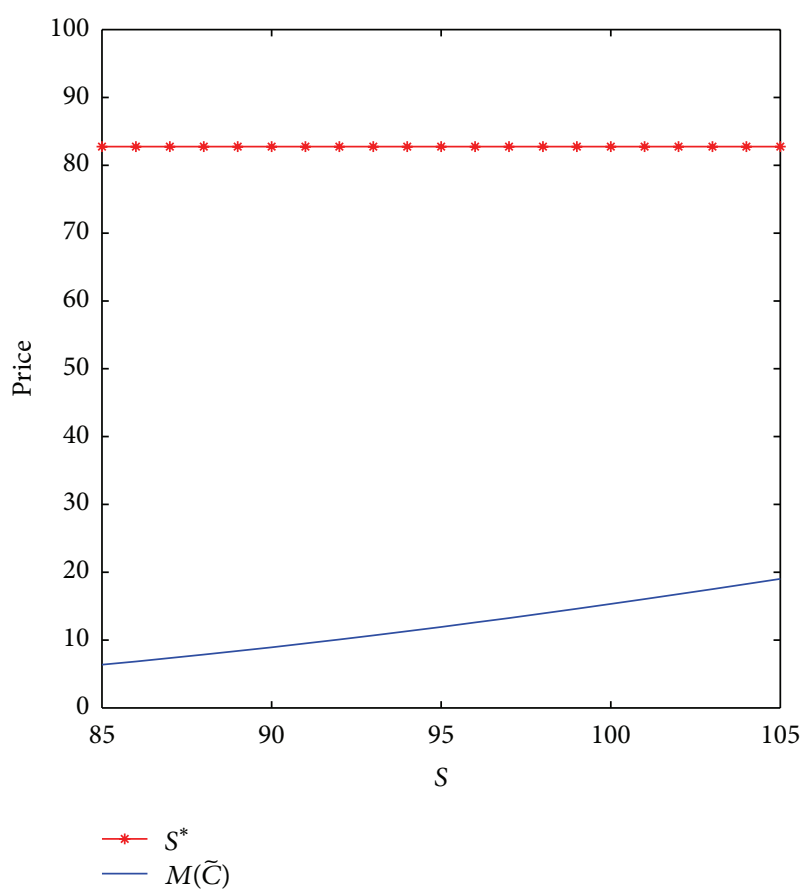

FIgURE 1: The sensitivity analysis of $S^{*}$ and $M(\widetilde{C})$ with respect to $S$.

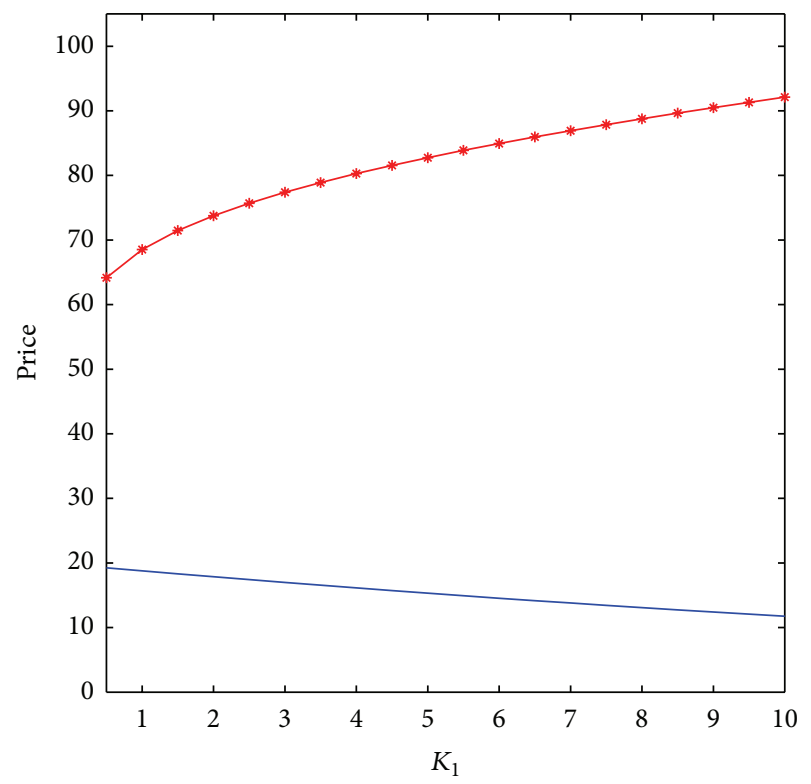

$$
\rightarrow S^{*}
$$$$
-M(\widetilde{C})
$$

FIGURE 2: The sensitivity analysis of $S^{*}$ and $M(\widetilde{C})$ with respect to $K_{1}$.

asset price at time $T_{1}$ such that the value of call option with the strike price $K_{2}$ and maturity time $T_{2}-T_{1}$ is equal to $K_{1}$; therefore, when $K_{1}$ is fixed (i.e., Figures 1, 3, 4, 5, 6, and 7), $S^{*}$ is increasing with respect to $K_{2}$ and $T_{1}$ and is decreasing with respect to $T_{2}, r_{c}$, and $\sigma_{c}$; when $K_{2}, T_{1}, T_{2}, r_{c}$, and $\sigma_{c}$ are fixed (i.e., Figure 2), $S^{*}$ is an increasing function with respect to $K_{1}$.

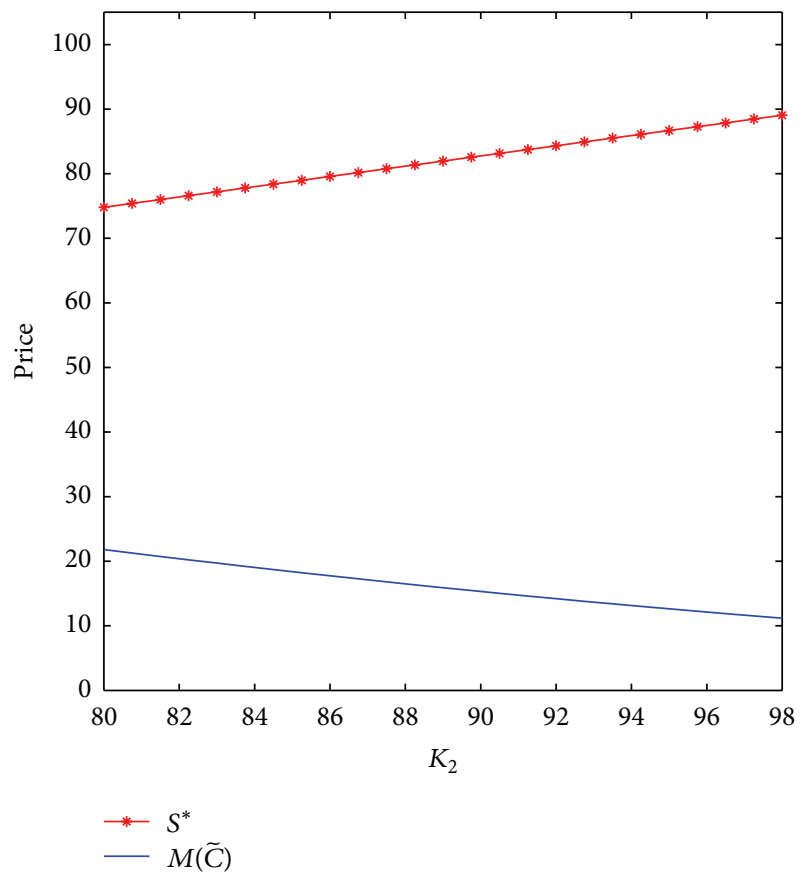

FIGURE 3: The sensitivity analysis of $S^{*}$ and $M(\widetilde{C})$ with respect to $K_{2}$.

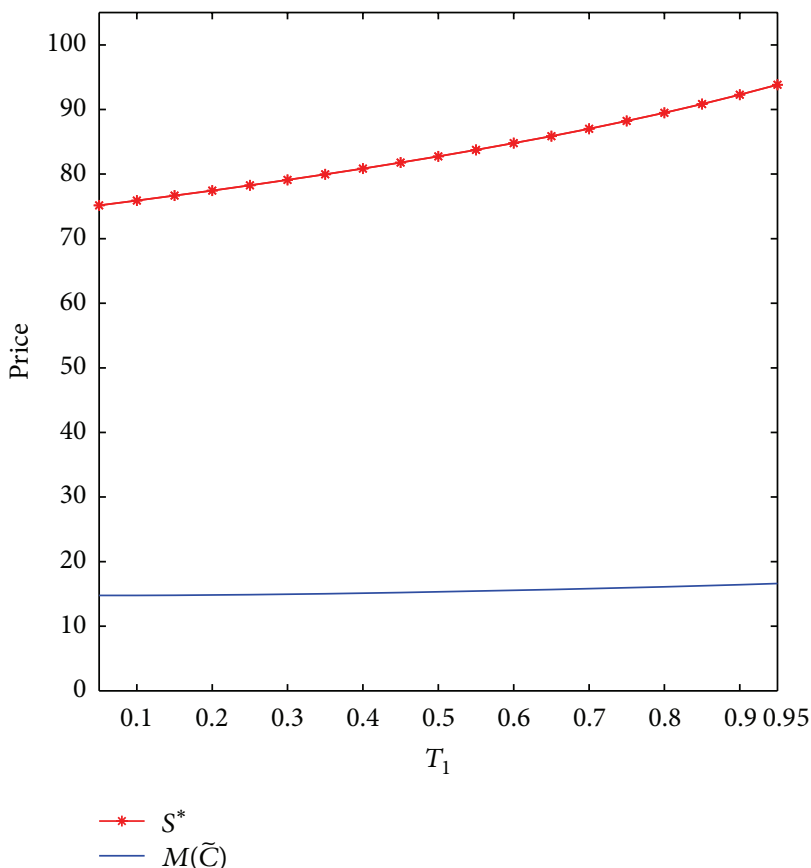

FIGURE 4: The sensitivity analysis of $S^{*}$ and $M(\widetilde{C})$ with respect to $T_{1}$.

We can see from Figures 1 to 7 that $S, T_{1}, T_{2}, r_{c}$, and $\sigma_{c}$ increase the value of $M(\widetilde{C})$ increases, and $K_{1}$ and $K_{2}$ increase the value of $M(\widetilde{C})$ decreases. This is consistent with $[1,7]$ that the compound option price is an increasing function with respect to $S, T_{1}, T_{2}, r$, and $\sigma$ and is a decreasing function with respect to $K_{1}$ and $K_{2}$. 


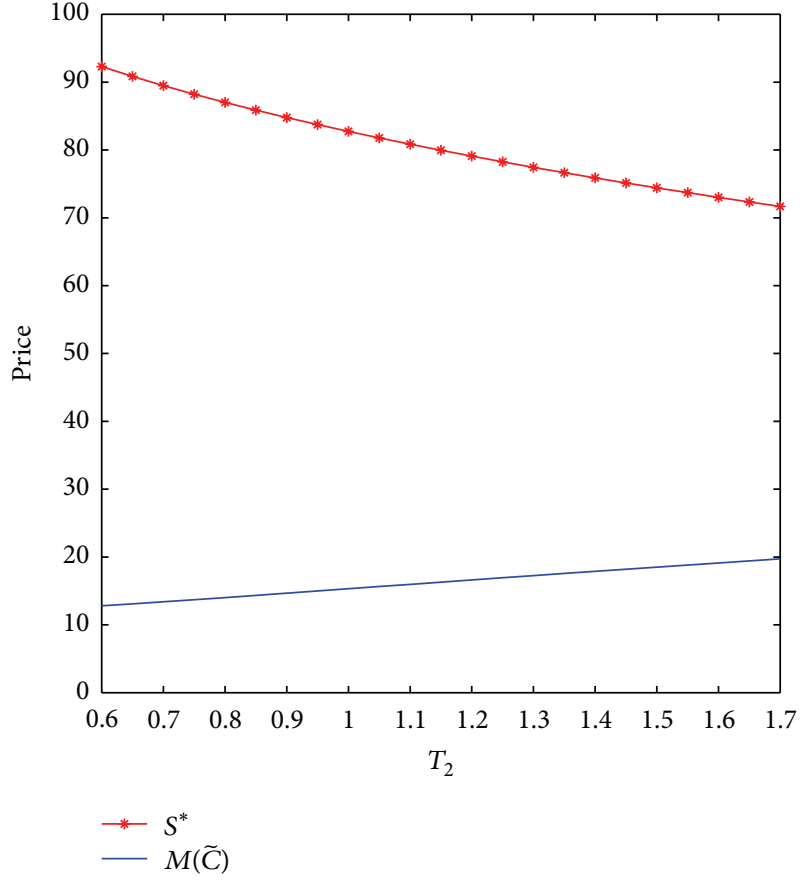

FIgURE 5: The sensitivity analysis of $S^{*}$ and $M(\widetilde{C})$ with respect to $T_{2}$.

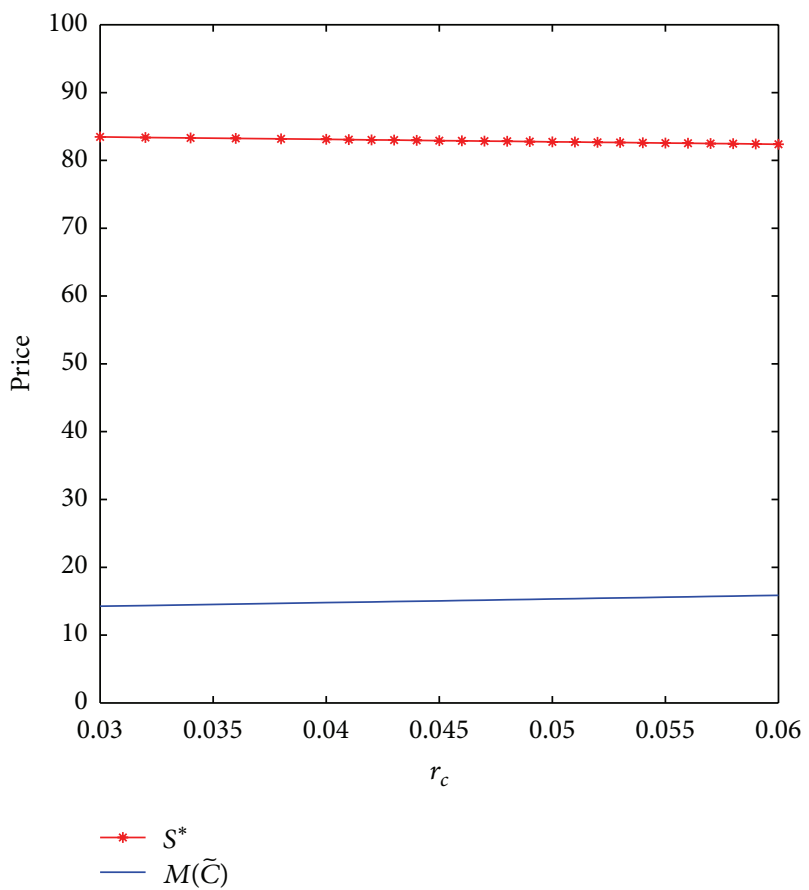

Figure 6: The sensitivity analysis of $S^{*}$ and $M(\widetilde{C})$ with respect to $r_{c}$.

Table 3 presents the closed interval of $\alpha$-level set of $\widetilde{C}$ calculated from Theorem 5 with $S=100, K_{1}=5, K_{2}=$ $90, T_{1}=0.5, T_{2}=1, \widetilde{r}=(0.048,0.05,0.051)$, and $\widetilde{\sigma}=$ $(0.29,0.3,0.32)$.

From Table 3, for $\alpha=0.95$, it means that the option price will lie in the closed interval [15.0977, 15.4649] with belief degree 0.95 . This interval can provide reference for financial

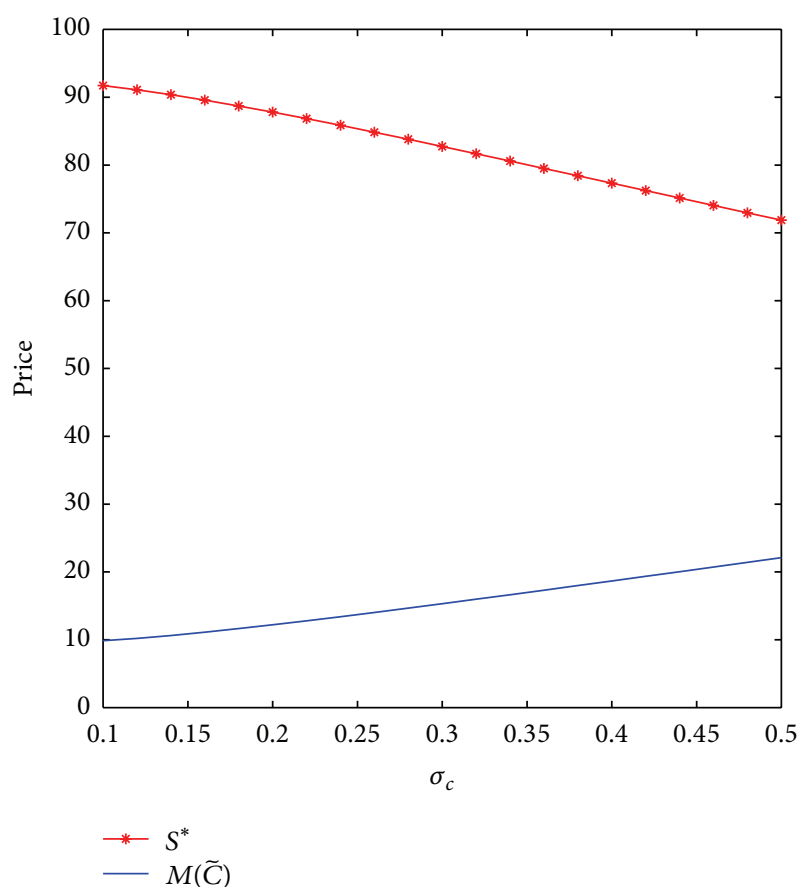

Figure 7: The sensitivity analysis of $S^{*}$ and $M(\widetilde{C})$ with respect to $\sigma_{c}$.

TABLE 3: The closed interval of $\alpha$-level set of $\widetilde{C}$.

\begin{tabular}{lc}
\hline$\alpha$ & Interval for fuzzy price \\
\hline 0.90 & {$[14.9214,15.6552]$} \\
0.91 & {$[14.9566,15.6171]$} \\
0.92 & {$[14.9919,15.5791]$} \\
0.93 & {$[15.0271,15.5410]$} \\
0.94 & {$[15.0624,15.5029]$} \\
0.95 & {$[15.0977,15.4649]$} \\
0.96 & {$[15.1330,15.4268]$} \\
0.97 & {$[15.1683,15.3887]$} \\
0.98 & {$[15.2036,15.3506]$} \\
0.99 & {$[15.2390,15.3125]$} \\
\hline
\end{tabular}

investors. That is to say, if a financial investor is satisfied with belief degree 0.95 , then he can pick any value from the closed interval $[15.0977,15.4649]$ as the option price. In addition, the financial investor may think the price is overvalued if the actual price option is higher than 15.4649 and sell the options; on the contrary, he may think the price is undervalued if the actual price option is less than 15.0977 and buy the options. We also notice that the length of the interval tends to become smaller with the increasing of belief degree and the interval with smaller belief degree contains the interval with larger belief degree.

\section{Conclusions}

Considering the uncertainty of the financial market includes both risk and vagueness; the compound option price will turn into a fuzzy number. We presented the fuzzy pricing formula 
for compound option by fuzzing the interest and volatility in Geske's compound option pricing formula. For any belief degree, the closed interval of price is obtained according to the fuzzy arithmetics and the definition of fuzzy-valued function; this allows the financial investor to make more flexible investment decision. We applied a defuzzification method to obtain the crisp possibilistic mean value of compound option price. Numerical analysis and sensitivity analysis showed that the crisp possibilistic mean value of compound option price is increasing with respect to the core of fuzzy interest rate and fuzzy volatility. The research on option pricing under fuzzy environment is a necessary improvement and supplement for the existing option pricing theory under stochastic model. In the future, we will study the compound option pricing under fuzzy environment based on jump-diffusion model, stochastic interest rate model, or stochastic volatility model and their applications.

\section{Conflict of Interests}

The authors declare that there is no conflict of interests regarding the publication of this paper.

\section{Acknowledgments}

This research is supported by the Natural Science Foundation of China (71071034 and 71201023) and the Funding of Jiangsu Innovation Program for Graduate Education (CXZZ 130140). The authors sincerely thank the anonymous referees for their helpful comments.

\section{References}

[1] R. Geske, "The valuation of compound options," Journal of Financial Economics, vol. 7, no. 1, pp. 63-81, 1979.

[2] F. Lajeri-Chaherli, "A note on the valuation of compound options," Journal of Futures Markets, vol. 22, no. 11, pp. 1103-1115, 2002.

[3] W. T. Lin, "Computing a multivariate normal integral for valuing compound real options," Review of Quantitative Finance and Accounting, vol. 18, no. 2, pp. 185-209, 2002.

[4] L. Thomassen and M. van Wouwe, "The n-fold compound option," Research Paper 2001-041, Department of Mathematics and Statistics, University of Antwerp, 2001.

[5] R. R. Chen, "The extended Geske-Johnson model and its consistency with reduced form models," Working Paper, Rutgers Business School, Rutgers University, 2003.

[6] M.-Y. Lee, F.-B. Yeh, and A.-P. Chen, "The generalized sequential compound options pricing and sensitivity analysis," Mathematical Social Sciences, vol. 55, no. 1, pp. 38-54, 2008.

[7] E. Agliardi and R. Agliardi, "A generalization of Geske formula for compound options," Mathematical Social Sciences, vol. 45, no. 1, pp. 75-82, 2003.

[8] E. Agliardi and R. Agliardi, "A closed-form solution for multicompound options," Risk Letters, vol. 1, no. 2, pp. 1-2, 2005.

[9] J.-P. Fouque and C.-H. Han, "Evaluation of compound options using perturbation approximation," Journal of Computational Finance, vol. 9, no. 1, pp. 41-61, 2005.
[10] C. R. Gukhal, "The compound option approach to American options on jump-diffusions," Journal of Economic Dynamics \& Control, vol. 28, no. 10, pp. 2055-2074, 2004.

[11] C. Chiarella and B. Kang, "The evaluation of American compound option prices under stochastic volatility and stochastic interest rates," The Journal of Computational Finance, vol. 14, no. 9, pp. 1-21, 2011.

[12] S. A. Griebsch, "The evaluation of European compound option prices under stochastic volatility using Fourier transform techniques," Review of Derivatives Research, vol. 16, no. 2, pp. 135165, 2013.

[13] R. Geske and H. E. Johnson, "The American put option valued analytically," The Journal of Finance, vol. 39, no. 5, pp. 1511-1524, 1984.

[14] P. Carr, "The valuation of sequential exchange opportunities," The Journal of Finance, vol. 43, no. 5, pp. 1235-1256, 1988.

[15] D. A. Paxson, "Sequential American exchange property options," The Journal of Real Estate Finance and Economics, vol. 34, no. 1, pp. 135-157, 2007.

[16] D. Cassimon, P. J. Engelen, L. Thomassen, and M. van Wouwe, "The valuation of a NDA using a 6-fold compound option," Research Policy, vol. 33, no. 1, pp. 41-51, 2004.

[17] Y.-L. Huang and C.-C. Pi, "Valuation of multi-stage BOT projects involving dedicated asset investments: a sequential compound option approach," Construction Management and Economics, vol. 27, no. 7, pp. 653-666, 2009.

[18] C. Park, J. M. Kang, and B. Min, "Compound real options incorporated with a stochastic approach for evaluating an uncertainty in petroleum exploration," Energy Sources B, vol. 8, no. 3, pp. 252-262, 2013.

[19] L. A. Zadeh, "Fuzzy sets," Information and Control, vol. 8, no. 3, pp. 338-353, 1965.

[20] Y. Yoshida, "The valuation of European options in uncertain environment," European Journal of Operational Research, vol. 145, no. 1, pp. 221-229, 2003.

[21] H.-C. Wu, "Pricing European options based on the fuzzy pattern of Black-Scholes formula," Computers and Operations Research, vol. 31, no. 7, pp. 1069-1081, 2004.

[22] K. A. Chrysafis and B. K. Papadopoulos, "On theoretical pricing of options with fuzzy estimators," Journal of Computational and Applied Mathematics, vol. 223, no. 2, pp. 552-566, 2009.

[23] M. L. Guerra, L. Sorini, and L. Stefanini, "Option price sensitivities through fuzzy numbers," Computers \& Mathematics with Applications, vol. 61, no. 3, pp. 515-526, 2011.

[24] A. Thavaneswaran, S. S. Appadoo, and A. Paseka, "Weighted possibilistic moments of fuzzy numbers with applications to GARCH modeling and option pricing," Mathematical and Computer Modelling, vol. 49, no. 1-2, pp. 352-368, 2009.

[25] Y. Yoshida, M. Yasuda, J. Nakagami, and M. Kurano, "A new evaluation of mean value for fuzzy numbers and its application to American put option under uncertainty," Fuzzy Sets and Systems, vol. 157, no. 19, pp. 2614-2626, 2006.

[26] Z. Změskal, "Generalised soft binomial American real option pricing model (fuzzy-stochastic approach)," European Journal of Operational Research, vol. 207, no. 2, pp. 1096-1103, 2010.

[27] A. Thavaneswaran, S. S. Appadoo, and J. Frank, "Binary option pricing using fuzzy numbers," Applied Mathematics Letters, vol. 26, no. 1, pp. 65-72, 2013.

[28] W. D. Xu, C. F. Wu, W. J. Xu, and H. Y. Li, "A jump-diffusion model for option pricing under fuzzy environments," Insurance: Mathematics and Economics, vol. 44, no. 3, pp. 337-344, 2009. 
[29] P. Nowak and M. Romaniuk, "Computing option price for Lévy process with fuzzy parameters," European Journal of Operational Research, vol. 201, no. 1, pp. 206-210, 2010.

[30] L. H. Zhang, W. G. Zhang, W. J. Xu, and W. L. Xiao, "The double exponential jump diffusion model for pricing European options under fuzzy environments," Economic Modelling, vol. 29, no. 3, pp. 780-786, 2012.

[31] H.-C. Wu, "Using fuzzy sets theory and Black-Scholes formula to generate pricing boundaries of European options," Applied Mathematics and Computation, vol. 185, no. 1, pp. 136-146, 2007.

[32] R. Fullér and P. Majlender, "On weighted possibilistic mean and variance of fuzzy numbers," Fuzzy Sets and Systems, vol. 136, no. 3, pp. 363-374, 2003. 


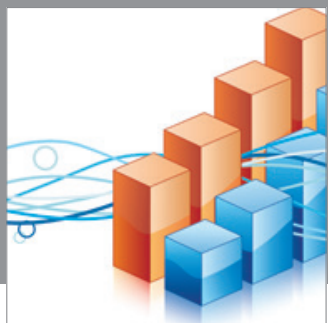

Advances in

Operations Research

mansans

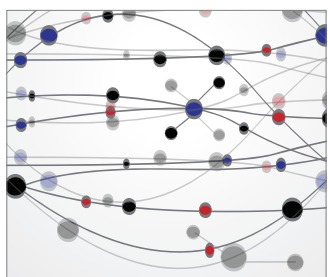

The Scientific World Journal
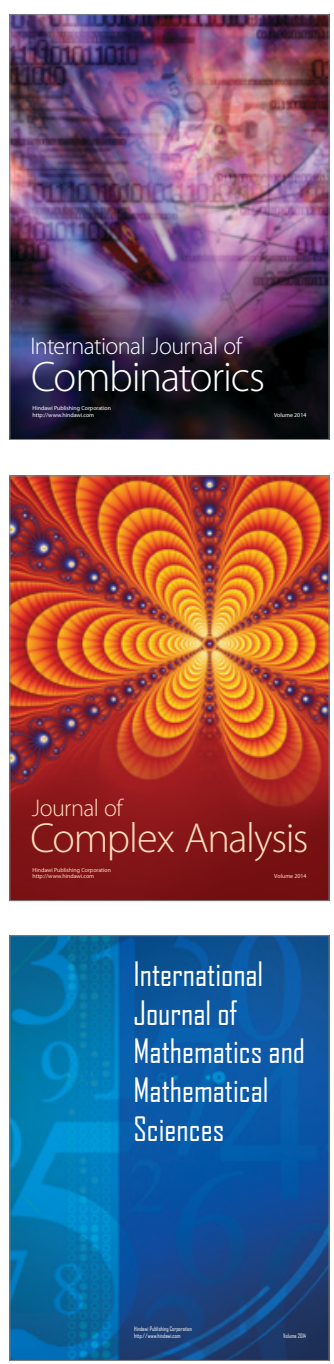
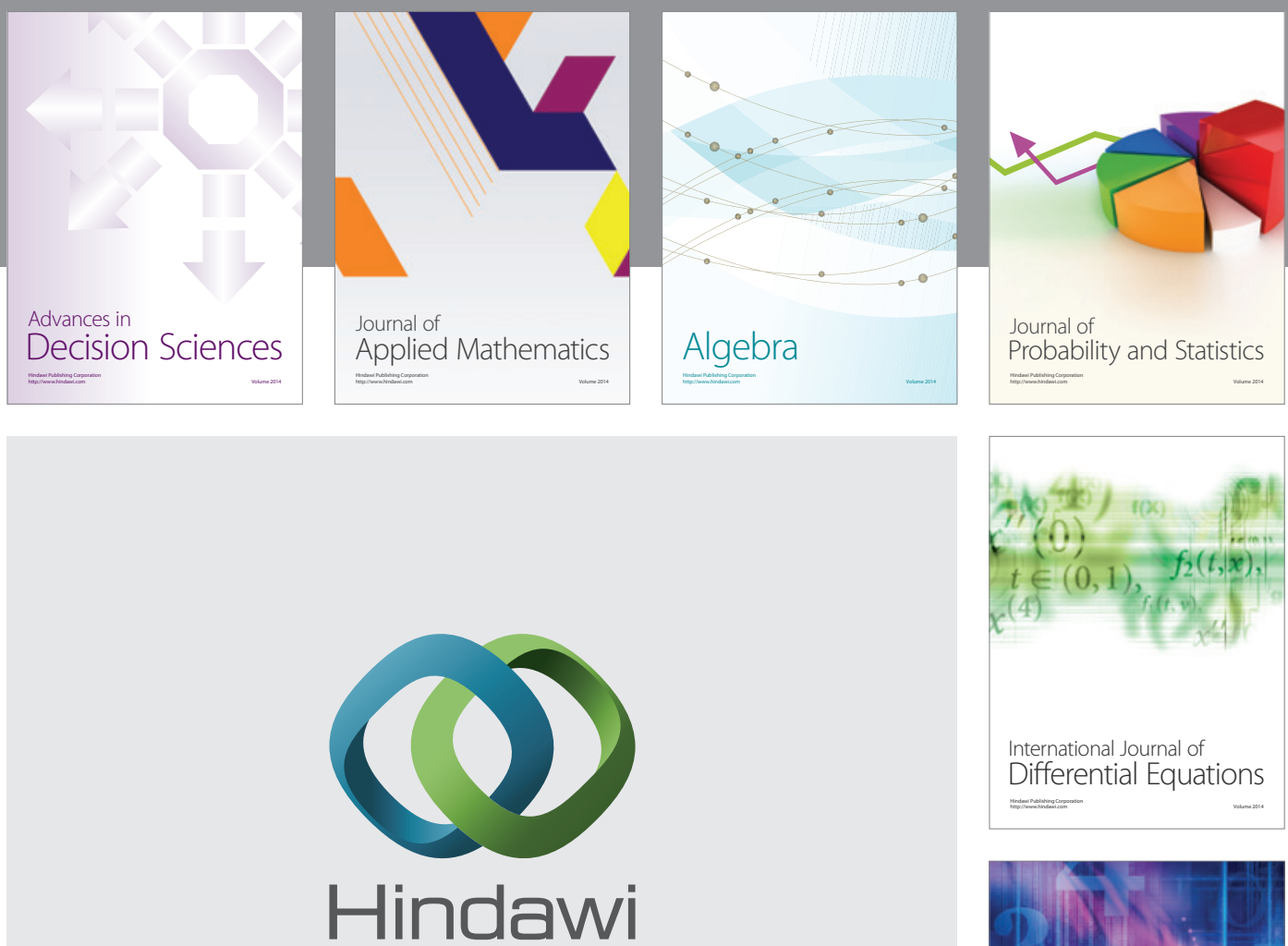

Submit your manuscripts at http://www.hindawi.com
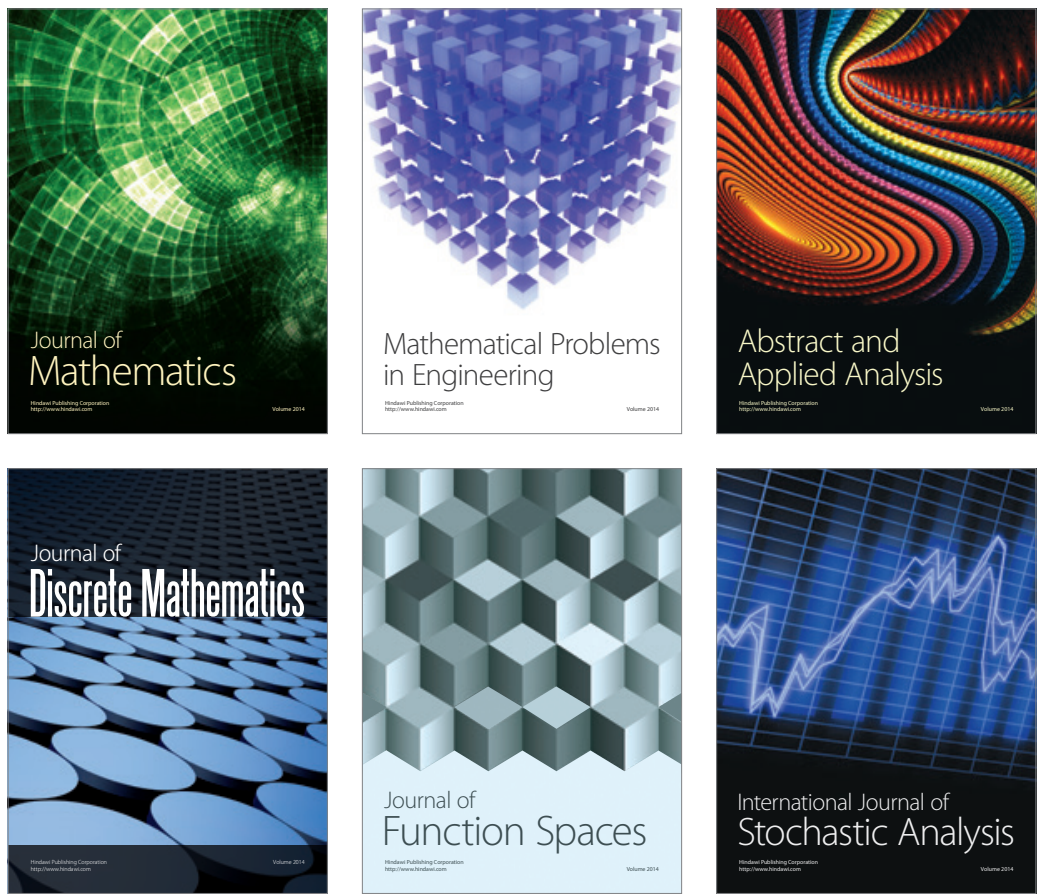

Journal of

Function Spaces

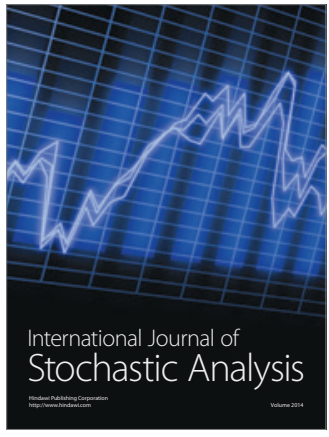

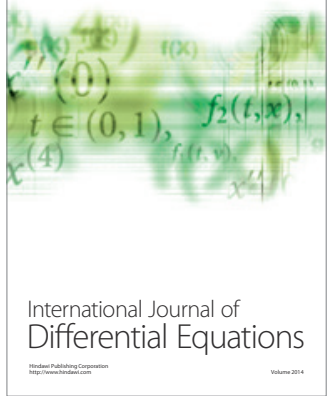
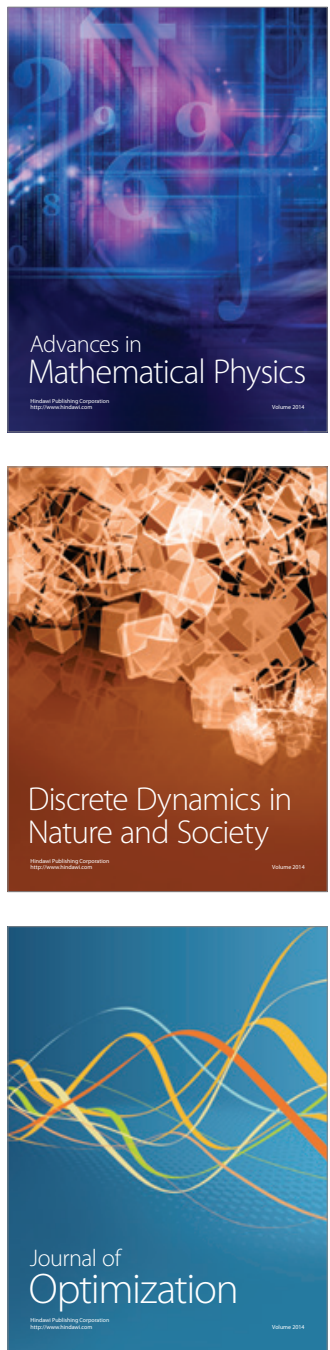